\title{
Recurrent atrial arrhythmia in a randomized controlled trial comparing contact force guided and contact force blinded ablation for typical atrial flutter
}

\author{
Mikkel Giehm-Reese ${ }^{1}$, Mads Kronborg ${ }^{1}$, Peter Lukac ${ }^{1}$, Steen Kristiansen ${ }^{1}$, Henrik Kjærulf \\ Jensen $^{1}$, Christian Gerdes ${ }^{1}$, Jens Kristensen ${ }^{1}$, Jan Møller Nielsen ${ }^{1}$, and Jens Nielsen ${ }^{1}$ \\ ${ }^{1}$ Aarhus University Hospital
}

October 29, 2021

\begin{abstract}
Background: Contact force (CF) guided catheter ablation (CA) is a novel technology developed to improve efficacy and reduce complications. In a randomised controlled trial $(\mathrm{RCT})$, we previously documented that after three months, rate of persistent conduction block was similar with and without using CF while performing CA for typical atrial flutter (AFL). Clinical effect of $\mathrm{CF}$ on recurrent arrhythmia is unknown. Objective: To study recurrent atrial arrhythmia during 12-months follow-up in a RCT investigating whether CF-guided CA for typical AFL is superior to CF-blinded CA. Method: Patients were randomized 1:1 to CA guided by CF (intervention group) or blinded to CF (control group). After 12 months, patients attended clinical check-up preceded by a 5-day ambulatory Holter monitor recording. Primary outcome was any recurrent atrial arrhythmia [?]30 seconds within 12 months, symptomatic or asymptomatic and documented in 12-lead ECG or Holter monitor recording. We did intention-to-treat (ITT) analysis. Results: We included and randomized 156 patients, four patients withdrew consent and two died during follow-up. Thus, 150 patients were included in ITT-analysis, in which recurrent arrhythmia was detected in $47(31 \%)$ patients, 25 in the intervention group and 22 in the control group $(\mathrm{p}=0.25)$. Atrial fibrillation was detected in 38 patients (18 versus 20 patients), and AFL in the remaining 9 patients ( 7 versus 2 patients). Conclusion: Contact force guided ablation for typical atrial flutter does not reduce recurrent atrial arrhythmia after 12-months follow-up as compared with ablation blinded for contact force.
\end{abstract}

Recurrent atrial arrhythmia in a randomized controlled trial comparing contact force guided and contact force blinded ablation for typical atrial flutter

Mikkel Giehm-Reese ${ }^{1}$, Mads Brix Kronborg ${ }^{1}$, Peter Lukac $^{1}$, Steen Buus Kristiansen ${ }^{1}$, Henrik Kjærulf Jensen $^{1}$, Christian Gerdes ${ }^{1}$, Jens Kristensen ${ }^{1}$, Jan Møller Nielsen ${ }^{1}$, Jens Cosedis Nielsen ${ }^{1,2}$

${ }^{1}$ Department of Cardiology, Aarhus University Hospital, Aarhus, Denmark, ${ }^{2}$ Department of Clinical Medicine, Aarhus University, Aarhus, Denmark

\section{Institution}

Department of Cardiology, Aarhus University Hospital, Aarhus, Denmark

Palle Juul-Jensens Boulevard 99

DK-8200 Aarhus N

Address for correspondence

Name: Mikkel Giehm-Reese 
Address: Palle Juul-Jensens Boulevard 99

Zip code and town: DK-8200 Aarhus N

Telephone: +4578456241

Email: mikkelgr@clin.au.dk

\section{Biographical notes on contributors}

Mikkel Giehm-Reese, MD, PhD, Aarhus University Hospital, Denmark.

Mads Brix Kronborg, MD, PhD, Consultant in Cardiology and electrophysiology, Aarhus University Hospital, Denmark.

Peter Lukac, MD, PhD, Consultant in Cardiology and electrophysiology, Aarhus University Hospital, Denmark.

Steen Buus Kristiansen, MD, PhD, Consultant in Cardiology and electrophysiology, Aarhus University Hospital, Denmark.

Henrik Kjærulf Jensen , MD, PhD, DMsc, Professor and consultant in cardiology and electrophysiology, Aarhus University Hospital, Denmark.

Christian Gerdes , MD, PhD, Consultant in Cardiology and electrophysiology, Aarhus University Hospital, Denmark.

Jens Kristensen, MD, PhD, Consultant in Cardiology and electrophysiology, Aarhus University Hospital, Denmark.

Jan Møller Jensen, MD, PhD, Consultant in Cardiology and electrophysiology, Aarhus University Hospital, Denmark.

Jens Cosedis Nielsen, MD, PhD, DMsc, Professor of cardiology, Department of Clinical Medicine, Aarhus University and Department of Cardiology, Aarhus University Hospital, Aarhus, Denmark.

\section{Funding}

This study was funded by an unrestricted institutional grant from Abbott.

\section{Conflict of interest}

M.G.R has received travel fees from Abbott. J.C.N is supported by an unrestricted grant from the Novo Nordisk Foundation (NNF16OC0018658). H.K.J is supported by an unrestricted grant from the Novo Nordisk Foundation (NNF18OC0031258) and has received lecturing fees from Abbott and Biosense Webster. C.G has received lecturing fees from Novartis. Consulting fees from Abbott, Medtronic, and Boehringer Ingelheim. Proctoring fees from Biosense Webster. All other authors declare no conflict of interest.

\section{Data availability statement}

The data underlying this article cannot be shared publicly due the privacy of the individuals included in the study.

\section{Abstract:}

Background: Contact force $(\mathrm{CF})$ guided catheter ablation $(\mathrm{CA})$ is a novel technology developed to improve efficacy and reduce complications. In a randomised controlled trial (RCT), we previously documented that after three months, rate of persistent conduction block was similar with and without using CF while performing $\mathrm{CA}$ for typical atrial flutter (AFL). Clinical effect of CF on recurrent arrhythmia is unknown.

Objective: To study recurrent atrial arrhythmia during 12-months follow-up in a RCT investigating whether CF-guided CA for typical AFL is superior to CF-blinded CA. 
Method: Patients were randomized 1:1 to CA guided by CF (intervention group) or blinded to CF (control group). After 12 months, patients attended clinical check-up preceded by a 5-day ambulatory Holter monitor recording. Primary outcome was any recurrent atrial arrhythmia [?]30 seconds within 12 months, symptomatic or asymptomatic and documented in 12-lead ECG or Holter monitor recording. We did intentionto-treat (ITT) analysis.

Results: We included and randomized 156 patients, four patients withdrew consent and two died during follow-up. Thus, 150 patients were included in ITT-analysis, in which recurrent arrhythmia was detected in $47(31 \%)$ patients, 25 in the intervention group and 22 in the control group $(\mathrm{p}=0.25)$. Atrial fibrillation was detected in 38 patients (18 versus 20 patients), and AFL in the remaining 9 patients ( 7 versus 2 patients).

Conclusion: Contact force guided ablation for typical atrial flutter does not reduce recurrent atrial arrhythmia after 12-months follow-up as compared with ablation blinded for contact force.

Keywords: Catheter ablation, typical atrial flutter, recurrent arrhythmia, contact force, lesion size index.

\section{Introduction:}

Cavo-tricuspid isthmus ablation (CTIA) using radiofrequency (RF) energy is a widely accepted first-line therapy for symptomatic typical atrial flutter (AFL) (1). Acute success rate is high, and risk of recurrent AFL is approximately $10 \%$ during long-term follow-up (FU) (2). Contact force (CF) guided catheter ablation (CA) has been proposed to improve acute procedural success when performing CA for atrial fibrillation (AF) $(3,4)$. However, data attained from randomised controlled trials (RCT) failed to show superiority for CFguided $\mathrm{CA}$ for $\mathrm{AF}$ compared to standard $\mathrm{CA}$ with regards to acute procedural success, complications and clinical outcome (5). In the setting of performing CF-guided CTIA, majority of studies conducted have been observational by design (6-8) and mainly focused on acute procedural parameters. Two RCT's found no difference in acute procedural parameters comparing CF-guided and CF-blinded CTIA $(9,10)$. In our recent $\mathrm{RCT}$, we found no difference regarding rate of cavo-tricuspid isthmus re-conduction at three months between CF-guided and CF-blinded CTIA (10). Whether long-term success from CF-guided CTIA is superior to CFblinded CTIA with respect to clinical outcome is unknown. The aim of this study is to present the 12-months results regarding recurrent atrial arrhythmia from our RCT comparing CF-guided and CF-blinded CTIA.

\section{Methods:}

Study design and population:

The study design has previously been described in detail (10). In short, this was a randomised, controlled, double-blinded, superiority single-centre study. We included patients with typical atrial flutter (AFL) referred for first-time cavo-tricuspid isthmus ablation (CTIA) at our institution. Exclusion criteria were congenital heart disease, atrial fibrillation $(\mathrm{AF})$ as the dominant arrhythmia, prior right atrial atriotomy, significant mitral valve disease, heart failure classified as New York Heart Association Class IV and secondary AFL. Before inclusion, all patients provided written informed consent. Patients were included between November 2016 and March 2019 at Department of Cardiology, Aarhus University Hospital, Denmark, and FU for the last patient ended in May 2020. After inclusion, patients were randomised in a 1:1 manner. In theintervention group, patients received CTIA guided by real-time contact force (CF) monitoring targeting pre-specified Lesion Size Index (LSI) value of 7.0. In the control group operators were blinded to real-time CF monitoring while performing CTIA. Patients were unaware of which group they were allocated to. The randomization tool used was a web-based case-record form (CRF) using computerized permuted blocks of different sizes. An external data manager was responsible for the CRF and had programmed the computerized random-number generator stratifying randomization according to gender. This study was approved by the local ethics committee and conforms to the declaration of Helsinki and is registered at www.clinicaltrials.gov (NCT02917538).

Cavo-tricuspid isthmus ablation:

All procedures were performed by experienced electrophysiologists. Patients were under conscious sedation, 
using fentanyl and midazolam. Catheterization of the patient was performed through the right femoral vein, placing a deflectable catheter in the coronary sinus with its proximal pole located at the coronary sinus ostium.

If a patient presented in AFL, entrainment was performed to ensure that the re-entrant mechanism of the flutter was CTI-dependent. Linear ablation was performed in all patients using point-by-point technique, creating an ablation line from the tricuspid annulus to the inferior vena cava (IVC). Power was set to $40 \mathrm{~W}$, irrigation of $17 \mathrm{ml}$ saline/min, and a target inter-lesion distance [?]5 mm using EnSite NavX 3D-mapping system (Abbott, Inc., IL, USA), TactiCath ${ }^{\mathrm{TM}}$ Quartz ablation catheter (Abbott, Inc., IL, USA), and an Agilis $^{\text {TM }}$ deflectable sheath (Abbott, Inc., IL, USA). In the intervention group, ablation was performed guided by real-time CF-monitoring targeting a specific LSI value of 7 . In the control group, operators were blinded to real-time $\mathrm{CF}$ values. Instead, they opted for one or more of the following: minimum 5-ohm impedance drop, $50 \%$ attenuation in EGM amplitude, and/or development of local split potentials. In case bidirectional isthmus block (BDIB) was not achieved by completion of the ablation line, additional ablation was performed. To ensure BDIB was achieved, standard differential pacing was performed. When BDIB persisted after a 10 min waiting period, the procedure was considered successful. Patients were discharged four hours after the procedure if no complications occurred, per our institutions' standard procedure.

Follow-up:

Patients were followed for a total of 12 months. Patients had previously received a 5-day ambulatory Holter monitor recording one month after their index procedure and undergone an invasive electrophysiological study (EPS) three months after their index procedure, as described earlier (10). At 12 months, patients attended clinical follow-up where a 12-lead ECG was recorded and preceded by a 5-day ambulatory Holter monitor recording. In cases where patients did not attend clinical follow-up or underwent 5-day ambulatory Holter-monitor recording, we were able to monitor clinical AF or AFL recurrence through their medical records.

Study outcomes:

The primary outcome of this study was recurrent arrhythmia at 12 months, defined as an episode of AFL or AF lasting at least 30 seconds, recorded in either 12-lead electrocardiogram (ECG), 5-day Holter-monitor recording or described in their medical records as being present on an ECG or a Holter-monitor recording (obtained in another institution) during FU. Major secondary outcomes were recurrent AFL, recurrent $\mathrm{AF}$, recurrent symptomatic AFL and AF, number of patients receiving re-ablation for either AFL or AF., recurrent arrhythmia according to reconduction status, and number of patients continuing oral anticoagulant (OAC) therapy. Furthermore, difference in age and $\mathrm{CHA}_{2} \mathrm{DS}_{2}$-VASc score for patients continuing and discontinuing OAC were analysed.

Statistical analysis:

Sample size calculation for this study has been described previously (10). Analysis was intention-to-treat (ITT). For the primary endpoint we performed a sensitivity analysis including patients adhering to the scheduled follow-up program (12-month clinical follow-up and received 5-day ambulatory Holter monitor recording). Parametric continuous data are presented as mean +- standard deviation (SD) and analysed using Student's t-test. Non-parametric continuous data are presented as medians with corresponding interquartile range (IQR) or range and analysed using Mann-Whitney U-test. Comparisons of multiple groups were performed using Kruskal-Wallis test or $\chi^{2}$ test. Categorical data were tested with either $\chi^{2}$ test or Fisher's exact test and presented as absolute numbers and frequencies. Ordinal data were analysed using Wilcoxon rank test and presented as median-value and absolute numbers. Hazard ratios with $95 \%$ confidence intervals (CI) were calculated using Cox proportional hazard regression, both crude and adjusted for the prespecified variables age, gender and $\mathrm{CHA}_{2} \mathrm{DS}_{2}$-VAsc score (dichotomized $<2$ or [?]2). Reported p-values are two-sided and the significance level was set to a $p$-value [?] 0.05. We used STATA version 16.1 (StataCorp LP College Station, Texas, USA) for Microsoft Windows for statistical analyses and data management. 


\section{Results:}

Baseline characteristics:

156 patients were enrolled in this trial (Figure 1). Median age was 68 (IQR 61-74) years, and 120 (77\%) patients were male. Baseline characteristics are reported in Table 1. In the intervention group, patients were older, more were female and the $\mathrm{CHA}_{2} \mathrm{DS}_{2}-\mathrm{VAS}_{\mathrm{c}}$ score was higher as compared to the control group. During follow-up, two patients died, one patient experienced non-cardiac death seven months after and one died nine months after inclusion from an acute myocardial infarction. No patients suffered a stroke or a transient ischemic attack.

Recurrent arrhythmia:

At one year FU, four patients had withdrawn consent, leaving $150(96 \%)$ patients available for analysis according to ITT principle. In these patients, recurrent arrhythmia was detected in 47 (31\%) patients, 25 in the intervention group and 22 patients in control group $(\mathrm{p}=0.25)$ (Figure 2). Atrial fibrillation was the dominant recurrent arrhythmia compared to AFL with no differences between the two groups (Figure 2.). Majority of AF episodes were asymptomatic in contrast to recurrent AFL episodes (Figure 2). Symptomatic and asymptomatic AF and AFL did not differ between the intervention- and control groups (Figure 2). Of the seven patients who experienced symptomatic recurrent AFL, all underwent renewed EPS and received CA. Three of these patients were diagnosed with atypical, non-isthmus-dependent AFL. Six patients received AF-ablation during follow-up. When performing regression analysis using Cox proportional hazard, risk of recurrent arrhythmia was not significantly lower for patients in the intervention group with a HR of 0.91 (95\% CI 0.51-1.64) unadjusted and 0.85 (95\% CI 0.46-1.57) adjusted

Sensitivity analysis:

126 patients (81\%) adhered to the study protocol. Recurrent arrhythmia was detected in 38 (31\%) patients, 19 patients in each of the two treatment groups. Neither recurrent AF nor AFL differed between the two groups, nor did symptomatic or asymptomatic recurrences (Table 2). Using Cox proportional hazard, risk of recurrent arrhythmia was not significantly lower for patients in the intervention group with a HR of 0.85 (95\% CI 0.48-1.60) unadjusted and 0.85 (95\% CI 0.48-1.62) adjusted.

Recurrent arrhythmia according to reconduction:

As reported earlier, CTI re-conduction was detected in 30 patients during EPS at three months FU (10). Fourteen of these patients (47\%) experienced recurrent arrhythmia during FU. Eleven had AF and three AFL. Thirty-three patients $(28 \%)$ of the hundred and twenty patients without re-conduction experienced recurrent arrhythmia. Twenty-seven had AF and six had AFL. The difference in proportion of patients experiencing recurrent arrhythmia between the two groups was statistically significant $(\mathrm{p}=0.03)$ (Figure 3). Using Cox proportional hazard, the higher risk of recurrent arrhythmia in patients with verified reconduction failed statistical significance with a HR 1.90 (95\% CI 0.98-3.69) unadjusted and 1,91(95\% CI 0.98-3.72) adjusted.

Oral-anticoagulant therapy:

At baseline, 152 (97\%) patients where on oral-anticoagulant (OAC) therapy, $117(75 \%)$ on a novel anticoagulant (NOAC). At 12 months FU, $100(67 \%)$ of the 150 patients included in the final analysis where still on OAC, 81 (81\%) on NOAC. Patients continuing OAC were significantly older (median age 71 years (IQR 64-75 years) vs 63 years (IQR 56-70 years) $\mathrm{p}=0.0001$ ). Dividing age into three categories, this further elucidates the difference in age composition between patients continuing and discontinuing OAC (Figure 4.A). Comparing $\mathrm{CHA}_{2} \mathrm{DS}_{2}$-VASc score between patients continuing and discontinuing OAC, this too was significantly higher in patients continuing OAC (median $\mathrm{CHA}_{2} \mathrm{DS}_{2}$-VASc score 3 (IQR 2-4) vs 1 (IQR 0-2) p $=0.0001)$. Dichotomizing $\mathrm{CHA}_{2} \mathrm{DS}_{2}$-VASc score into $<2$ or [?]2, majority of patients continuing OAC had a $\mathrm{CHA}_{2} \mathrm{DS}_{2}$-VASc score [?]2 compared to patients discontinuing OAC (Figure 4.B).

\section{Discussion:}


In this single-centre randomized double-blinded trial including 156 patients with typical AFL, we showed that CTIA guided by real-time CF targeting a specific LSI value did not reduce recurrent atrial arrhythmia after 12 months. Recurrent AF was more common than AFL, and more often was asymptomatic. At the end of FU, we observed that $33 \%$ of patients discontinued OAC.

The long-term results on occurrence of clinical arrhythmias in our RCT are consistent with our previous findings, where no difference was found in cavo-tricuspid isthmus re-conduction at three months between the intervention and control groups (10). Our results thus show that CF-guided CTIA confers no benefit regarding acute procedural success, peri-procedural complications, re-conduction (10), or 12-months recurrent symptomatic or asymptomatic atrial arrhythmia. This despite CF- and LSI-values being higher for patents in the intervention group (10). Long-term results regarding recurrent arrhythmia following CF-guided CTIA are scarce. Comparing our results to those from RCT's investigating CF-guided CA for AF, conclusions are similar showing no added benefit from CF (5). This is in contrast to several observational and experimental studies, showing that CF-guided CA reduces procedure-, fluoroscopy, and ablation time as well as making more durable lesions $(6-8,11-16)$. However, the gold standard for testing effect of an intervention is the $\mathrm{RCT}$, and with respect to the effect of CF-guided CA, there is no doubt that data obtained in RCT's best reflects the true signal.

Ablation Index (B) ((AI), Biosense Webster Inc, CA, USA) is an alternative technology to LSI that has shown promising results for CTIA $(11,17)$. Especially, the large multi-centre observational study by Viola et al (17), enrolling 412 patients. However, this was a non-randomized trial with the primary aim of studying acute procedural efficacy and safety, not recurrent arrhythmia. To date, all studies evaluating the use of AI in both CTIA, and AF-ablation are of observational design as highlighted in a recent meta-analysis (18).

Nine (6\%) patients experienced recurrent AFL during FU. These results are in line with a previous large Danish observational study showing rate of recurrent AFL at $10 \%$ over a four-year FU period (2). Majority of these cases occurred within the first year. A large Swedish study found similar results (19). However, adding to explain the low rate of recurrent AFL may be additional CTIA at three months EPS in 30 patients with re-conduction until BDIB was reaffirmed. Some of these patients may eventually have developed AFL.

Thirty-eight (25\%) patients experienced AF during 12 months of follow-up. A Danish observational study reported a rate of almost 30\% of patients experiencing AF over a four-year FU period after CTIA (2). However, these were cases of AF leading to contact to the healthcare system and registered via the Danish National Patient Registry (DNPR), and thus not including many asymptomatic and paroxysmal cases. In contrast, the majority of $\mathrm{AF}$ cases in the current study were asymptomatic (26 of 38). It is worth mentioning, not all cases were new-onset AF, as $4 \%$ of patients had known AF prior to undergoing CTIA. It is well-known that with more intensive monitoring more $\mathrm{AF}$ will be detected $(20,21)$. Observations from our study highlights that AF following CTIA is more common than recurrent AFL (22, 23), and especially asymptomatic AF is frequent; and may likely be underestimated in every-day clinical practice. Thus, highlighting the importance of continuing OAC in high-risk patients even after successful CTIA.

Re-conduction found at three-month EPS was associated with increased risk of recurrent arrhythmia, both AFL and AF. Re-conduction across the CTIA-line being associated with recurrent AFL seems straightforward. Four of seven patients receiving renewed CA for their AFL, had typical AFL. The remaining three had atypical AFL. Whether these three patients initially had atypical AFL mimicking typical AFL or this was induced due to atrial scarring from CA or disease progression is uncertain. The association between CTI re-conduction and AF might be more complex, and the exact underlying mechanism cannot be determined from the present study. It is well-known that AFL and AF have an intertwined relationship, and AF is common after CTIA (2). Pre-existing AF has been proposed as a necessity for developing AFL (24).

Discontinuation of OAC was high in the present study, with $33 \%$ of patients having discontinued OAC at 12 months FU. A recent Danish nation-wide observational study found $30 \%$ of patients discontinuing OAC following CTIA over a four-year period (25). The study was a registry-based study, and incidence of discontinuing OAC might have been underestimated. In this study as well as the present, patients discontinuing 
OAC were significantly younger and had lower $\mathrm{CHA}_{2} \mathrm{DS}_{2}$-VASc scores significantly than patients continuing OAC. Although, success rate is high following CTIA regarding recurrent AFL, development of AF is a concern (2). Therefore, considering discontinuing OAC in patients following CTIA, both risk of AF and their thromboembolic risk should be cautiously considered.

Strengths and limitations:

The data presented in the current study originates from a double-blind RCT, which reduces the risk of treatment bias. An important limitation is that the study was originally powered to detect difference in re-conduction at three-month EPS and not difference in recurrent arrhythmia at 12 months. Nonetheless, these clinical results are in line with our previously published electrophysiological results (10), and therefore supports the main finding that CF-guided CTIA is not superior to CTIA without CF-guidance. Originally, 156 patients were included in the study. Only 126 (81\%) of these adhered strictly to the FU program specified in the study protocol. However, our findings are similar in both our ITT and sensitivity analyses indicating no effect modification of our results. In the intervention group, patients were slightly older, more were women, and they had a higher median $\mathrm{CHA}_{2} \mathrm{DS}_{2}-\mathrm{VAS}_{\mathrm{c}}$ score than patients in the control group. Risk of developing AF following CTIA has been associated with higher age and patient comorbidities $(2,23$, 26). These differences however were a result of chance at randomization. They may have contributed to a higher incidence of recurrent arrhythmia in the intervention group. This is a single-centre study, which offers a high level of homogeneity in performing CTIA procedures. Caution therefore should be taken before extrapolating these results into a broader setting. To best evaluate whether CF-guided CA is superior or not to CF-blinded CA, more research is needed. Preferably in the setting of large multi-centre RCT's evaluating recurrent arrhythmia and not acute procedural efficacy and safety endpoints primarily.

\section{Conclusion:}

Contact force-guided ablation for typical atrial flutter does not reduce recurrent arrhythmia after 12 months follow-up as compared with ablation blinded to contact force.

References

1. Lee G, Sanders P, Kalman JM. Catheter ablation of atrial arrhythmias: state of the art. The Lancet. 2012;380(9852):1509-19.

2. Giehm-Reese M, Kronborg MB, Lukac P, Kristiansen SB, Nielsen JM, Johannessen A, et al. Recurrent atrial flutter ablation and incidence of atrial fibrillation ablation after first-time ablation for typical atrial flutter: A nation-wide Danish cohort study. International Journal of Cardiology. 2020;298:44-51.

3. Kautzner J, Neuzil P, Lambert H, Peichl P, Petru J, Cihak R, et al. EFFICAS II: optimization of catheter contact force improves outcome of pulmonary vein isolation for paroxysmal atrial fibrillation. EP Europace. 2015;17(8):1229-35.

4. Reddy V, Dukkipati S, Neuzil P, Natale A, Albenque J, Kautzner J, et al. Randomized, Controlled Trial of the Safety and Effectiveness of a Contact Force-Sensing Irrigated Catheter for Ablation of Paroxysmal Atrial Fibrillation: Results of the TactiCath Contact Force Ablation Catheter Study for Atrial Fibrillation (TOCCASTAR) Study. Circulation. 2015;132(10):907-15.

5. Virk SA, Ariyaratnam J, Bennett RG, Kumar S. Updated systematic review and meta-analysis of the impact of contact force sensing on the safety and efficacy of atrial fibrillation ablation: discrepancy between observational studies and randomized control trial data. EP Europace. 2018;21(2):239-49.

6. Gould PA, Booth C, Dauber K, Ng K, Claughton A, Kaye GC. Characteristics of cavotricuspid isthmus ablation for atrial flutter guided by novel parameters using a contact force catheter. Journal of cardiovascular electrophysiology. 2016;27(12):1429-36.

7. Kumar S, Morton JB, Lee G, Halloran K, Kistler PM, Kalman JM. High Incidence of Low Catheter-Tissue Contact Force at the Cavotricuspid Isthmus During Catheter Ablation of Atrial Flutter: Implications for 
Achieving Isthmus Block. Journal of cardiovascular electrophysiology. 2015;26(8):826-31.

8. Venier S, Andrade JG, Khairy P, Mondesert B, Dyrda K, Rivard L, et al. Contact-force-guided vs. contactforce-blinded catheter ablation of typical atrial flutter: a prospective study. Ep Europace. 2016;19(6):1043-8.

9. Begg GA, O’Neill J, Sohaib A, McLean A, Pepper CB, Graham LN, et al. Multicentre randomised trial comparing contact force with electrical coupling index in atrial flutter ablation (VERISMART trial). PloS one. 2019;14(4):e0212903.

10. Giehm-Reese M, Kronborg MB, Lukac P, Kristiansen SB, Jensen HK, Gerdes C, et al. A randomized trial of contact force in atrial flutter ablation. EP Europace. 2020;22(6):947-55.

11. Zhang T, Wang Y, Han Z, Zhao H, Liang Z, Wang Y, et al. Cavotricuspid isthmus ablation using ablation index in typical right atrial flutter. Journal of Cardiovascular Electrophysiology. 2019;30(11):2414-9.

12. Whitaker J, Fish J, Harrison J, Chubb H, Williams SE, Fastl T, et al. Lesion Index-Guided Ablation Facilitates Continuous, Transmural, and Durable Lesions in a Porcine Recovery Model. Circulation: Arrhythmia and Electrophysiology. 2018;11(4):e005892.

13. Hussein A, Das M, Riva S, Morgan M, Ronayne C, Sahni A, et al. Use of Ablation Index-Guided Ablation Results in High Rates of Durable Pulmonary Vein Isolation and Freedom From Arrhythmia in Persistent Atrial Fibrillation Patients. Circulation: Arrhythmia and Electrophysiology. 2018;11(9):e006576.

14. Reddy VY, Shah D, Kautzner J, Schmidt B, Saoudi N, Herrera C, et al. The relationship between contact force and clinical outcome during radiofrequency catheter ablation of atrial fibrillation in the TOCCATA study. Heart rhythm. 2012;9(11):1789-95.

15. Neuzil P, Reddy VY, Kautzner J, Petru J, Wichterle D, Shah D, et al. Electrical Reconnection After Pulmonary Vein Isolation Is Contingent on Contact Force During Initial TreatmentClinical Perspective: Results From the EFFICAS I Study. Circulation: Arrhythmia and Electrophysiology. 2013;6(2):327-33.

16. Boles U, Gul EE, Fitzpatrick N, Enriquez A, Baranchuk A, Simpson C, et al. Lesion Size Index in Maximum Voltage-Guided Cavotricuspid Ablation for Atrial Flutter. The Journal of Innovations in Cardiac Rhythm Management, 8 (2017), 2732-2738. 2017;2733.

17. Viola G, Stabile G, Bandino S, Rossi L, Marrazzo N, Pecora D, et al. Safety, efficacy, and reproducibility of cavotricuspid isthmus ablation guided by the ablation index: acute results of the FLAI study. EP Europace. 2020.

18. Ioannou A, Papageorgiou N, Lim WY, Wongwarawipat T, Hunter RJ, Dhillon G, et al. Efficacy and safety of ablation index-guided catheter ablation for atrial fibrillation: an updated meta-analysis. EP Europace. 2020;22(11):1659-71.

19. Carlson J, Kongstad O, Holmqvist F, Ringborn M, Herczku C, Kesek M, et al. A decade of catheter ablation of cardiac arrhythmias in Sweden: ablation practices and outcomes. European Heart Journal. 2018;40(10):820-30.

20. Buck BH, Hill MD, Quinn FR, Butcher KS, Menon BK, Gulamhusein S, et al. Effect of Implantable vs Prolonged External Electrocardiographic Monitoring on Atrial Fibrillation Detection in Patients With Ischemic Stroke: The PER DIEM Randomized Clinical Trial. JAMA. 2021;325(21):2160-8.

21. Bernstein RA, Kamel H, Granger CB, Piccini JP, Sethi PP, Katz JM, et al. Effect of Long-term Continuous Cardiac Monitoring vs Usual Care on Detection of Atrial Fibrillation in Patients With Stroke Attributed to Large- or Small-Vessel Disease: The STROKE-AF Randomized Clinical Trial. JAMA. 2021;325(21):216977.

22. Celikyurt U, Knecht S, Kuehne M, Reichlin T, Muehl A, Spies F, et al. Incidence of new-onset atrial fibrillation after cavotricuspid isthmus ablation for atrial flutter. Ep Europace. 2017;19(11):1776-80. 
23. De Bortoli A, Shi L-B, Ohm O-J, Hoff PI, Schuster P, Solheim E, et al. Incidence and clinical predictors of subsequent atrial fibrillation requiring additional ablation after cavotricuspid isthmus ablation for typical atrial flutter. Scandinavian Cardiovascular Journal. 2017;51(3):123-8.

24. Waldo AL, Feld GK. Inter-relationships of atrial fibrillation and atrial flutter: mechanisms and clinical implications. Journal of the American College of Cardiology. 2008;51(8):779-86.

25. Giehm-Reese M, Johansen MN, Kronborg MB, Jensen HK, Gerdes C, Kristensen J, et al. Discontinuation of oral anticoagulation and risk of stroke and death after ablation for typical atrial flutter: A nation-wide Danish cohort study. International Journal of Cardiology. 2021;333:110-6.

26. Chen K, Bai R, Deng W, Gao C, Zhang J, Wang X, et al. HATCH score in the prediction of new-onset atrial fibrillation after catheter ablation of typical atrial flutter. Heart Rhythm. 2015;12(7):1483-9.

Table 1. Baseline characteristics.

All patients (n=156) Intervention group (n=79) Control group (n=77) Age (IQR)* 68 (61-74) 71 (62-75) 67 (57-

All patients $(\mathbf{n}=\mathbf{1 2 6})$ Intervention group $(\mathbf{n}=\mathbf{5 8})$ Control group $(\mathbf{n}=\mathbf{6 8})$ p-value Recurrent arrhythmia*, n $(\%) 38$

\section{Figure legends:}

Figure 1. Consort diagram depicting number of patients screened, randomised, and included in final analysis.

Figure 2. Sideways bar graphs displaying number of patients with both symptomatic and asymptomatic $\mathrm{AF}$ and AFL among intervention- and control group. Number of patients is shown numerically within each bar.

Figure 3. Sideways bar graphs displaying distribution of patients with no recurrent arrhythmia, AF and AFL at 12 months; among patients with and without cavo-tricuspid isthmus re-conduction at three-months EPS. Number of patients is shown numerically at the end of each bar.

Figure 4.A. Bar graph displaying difference in age between patients on and off OAC according to three different age categories. Number of patients shown atop of each bar. B. Pie graphs depicting number of patients with a $\mathrm{CHA}_{2} \mathrm{DS}_{2}$-VASc score of $0,1,2,3$ or [?] 4 for patients on and off OAC. Number of patients are shown numerically within each circle.

\section{Hosted file}

image1.emf available at https://authorea.com/users/443638/articles/543606-recurrent-atrialarrhythmia-in-a-randomized-controlled-trial-comparing-contact-force-guided-and-contactforce-blinded-ablation-for-typical-atrial-flutter

Figure 1.

\section{Hosted file}

image2.emf available at https://authorea.com/users/443638/articles/543606-recurrent-atrialarrhythmia-in-a-randomized-controlled-trial-comparing-contact-force-guided-and-contactforce-blinded-ablation-for-typical-atrial-flutter

Figure 2 .

Figure 3.

\section{Hosted file}


image3.emf available at https://authorea.com/users/443638/articles/543606-recurrent-atrialarrhythmia-in-a-randomized-controlled-trial-comparing-contact-force-guided-and-contactforce-blinded-ablation-for-typical-atrial-flutter

Figure 4 .

\section{Hosted file}

image4.emf available at https://authorea.com/users/443638/articles/543606-recurrent-atrialarrhythmia-in-a-randomized-controlled-trial-comparing-contact-force-guided-and-contactforce-blinded-ablation-for-typical-atrial-flutter 